\title{
Claudio Galeno y los ventrículos cerebrales. Parte I, los antecedentes
}

\author{
F. Martínez y G. Decuadro-Sáenz
}

Departamento de Anatomía. Facultad de Medicina de Montevideo. Uruguay.

\section{Resumen}

Claudio Galeno es considerado uno de los padres de la medicina occidental. Por el entorno cultural y religioso en el cual estaba inmerso, se duda de que haya disecado cadáveres y se piensa que la mayoría de sus aportes al conocimiento anatómico provengan del estudio de monos.

En este trabajo, los autores analizan la teoría anatomofuncional de Galeno sobre los ventrículos cerebrales. Dado que muchos de los conceptos galénicos provienen de sus antecesores ( $y$ en particular de la medicina hipocrática), la presentación del mismo se hace en dos partes. En primera instancia, se exponen los antecedentes anatómicos, religiosos y filosóficos que influyeron a Galeno. Este largo periodo de la historia está dividido a nuestros propósitos en tres etapas: 1) la era pretécnica, donde no hay un conocimiento rudimentario y fundamentalmente derivado de prácticas religiosas. Este periodo llega hasta el siglo VI AC. 2) la ciencia helenística, que abarca los siglos VI a III AC. En esta etapa se gesta y desarrolla la medicina hipocrática, y 3) el periodo que comprende el siglo III AC hasta Galeno.

En cada una de las eras descritas, se analizan y destacan los mayores aportes al conocimiento neuroanatómico de cada cultura.

PALABRAS CLAVE: Claudio Galeno. Ventrículos cerebrales. Anatomía. Historia de la Medicina.

Claudio Galeno and the lateral ventricles

\begin{abstract}
Galen of Pergamon is considered one of the fathers of occidental medicine. Because of cultural and religious precepts at his time, many authors hypothesize that the anatomical concepts of Galen were derived from dissec-
\end{abstract}

Recibido: 12-07-06. Aceptado: 10-10-06 tion of monkeys.

In this paper, the authors analyzed the ventricular system, according to the anatomic and functional concepts described by Galen's early work. Knowing that his work was derived and or influenced from other preceding anatomists and physicians (specially Hipocrates of Cos), we present our investigation in two parts. In the first part, we present the influences of previous religious, philosophic, medical and anatomical theories over the galenic work. The "pregalenic era" can be divided in three periods: 1) the pretecnic era (ending in VI BC) in wich the man have only a rudimentary knowledge of human anatomy, 2) the helenistic science (from VI to III BC) and 3) the period comprised between III BC to Galenic period.

In each of the three periods, we analyzed the neuroanatomic knowledge of the most important cultures.

KEY WORDS: Galen. Ventricular system. Anatomy. History of anatomy.

Introducción

"...En cuanto médico, ese hombre está convencido de vivir en un momento importante; pero no desconoce que su situación histórica tiene pasado, al cual debe su origen lo que él sabe, y un futuro, en el cual los médicos, si en su práctica siguen un método correcto, pueden llegar a saber más..."16.

Claudio Galeno es considerado uno de los padres de la medicina occidental. Planteó que la medicina debía apoyarse en bases anatómicas y fisiológicas surgidas de la observación, disección y experimentación: todo tenía un porqué que debía ser explicado ${ }^{2,16-18}$.

Sus trabajos sirvieron como base a anatomistas que re-describieron estructuras previamente observadas por él. Dejó un importante legado científico que influyó en médicos bizantinos, árabes, escolásticos y bajomedievales ${ }^{6,13-}$ $15,19,26,32,34$. Se conocieron en Europa más de 20 ediciones latinas de las obras completas de Galeno que fueron leídas 
y comentadas por la mayoría de los médicos hasta el siglo $\mathrm{XVI}^{18}$.

Sin embargo, Galeno acuño teorías, conceptos y descripciones anatómicas erróneas y si bien contó con detractores en todas las épocas, existía un gran respeto hacia sus obras, lo que perpetuó sus errores por más de mil años ${ }^{16,17}$.

Se duda que haya disecado cadáveres humanos de forma reglada, con lo que sus conocimientos anatómicos estarían basados fundamentalmente en disecciones de ani-

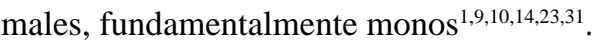

En el presente trabajo se estudia la concepción anatomofisiológica de Galeno sobre los ventrículos cerebrales a partir de una revisión bibliográfica. La obra que sirve de base para este artículo es Oeuvres anatomiques, physiologiques et médicales de Galien de Charles Daremberg ${ }^{7}$, la cuál es una traducción al francés de De usus partius de Galeno.

Este artículo se presenta en dos partes: en una primera instancia se verán los antecedentes anatómicos, religiosos, médicos y filosóficos en los cuales se apoyó Galeno para sus concepciones. Esto es imprescindible para comprender la segunda parte del trabajo, donde se verá específicamente la concepción galénica de los ventrículos cerebrales y sus influencias particulares.

\section{El conocimiento neuroanatómico previo a Galeno}

La evolución de la anatomía en la antigüedad puede agruparse en tres grandes etapas:

1) la era pretécnica, que abarca los inicios de una anatomía rudimentaria hasta el siglo VI AC.

2) la etapa de la antigua Grecia o ciencia helenística (entre los siglos VI y III AC).

3) el período comprendido entre el siglo III AC hasta Galeno, época donde se destaca el esplendor de la escuela de Alejandría.

\section{a) La era pretécnia (el azul antes del amanecer)}

El "conocimiento anatómico" es tan antiguo como la Medicina y el propio hombre, que observaba el interior de un compañero herido en una batalla o en la cacería de una fiera ${ }^{30}$. En las cuevas de Lascaux (Francia) y Altamira (España) hay pinturas rupestres de 14.000 a 17.000 años de antigüedad que muestran un búfalo herido con sus vísceras hacia el exterior. Las figuras humanas son representadas aquí de forma muy esquemática y no se pueden apreciar detalles en ellas.

Se han encontrado figuras humanas talladas, de 22.000 años de antigüedad en las áreas de las actuales Rusia y Checoslovaquia; pero no pueden verse detalles. También han sido encontradas representaciones de figuras humanas en culturas sudamericanas, norteamericanas y africanas, aunque no tan antiguas (Figura 1). Pero, no se puede con-
2008; 19: 58-65

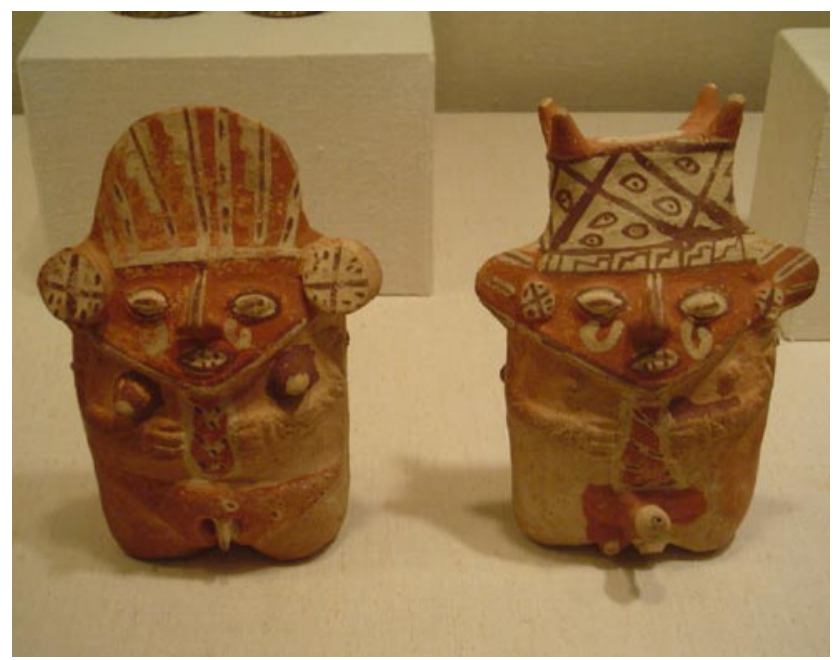

Figura 1. Representaciones de figuras humanas en diferentes culturas.

A. Figuras de arcilla correspondientes a culturas Sudamericanas. Obsérvese que el sector genital es el único bien desarrollado. Estas esculturas probablemente formaran parte de ofrendas, destacando los genitales como culto a la fertilidad.

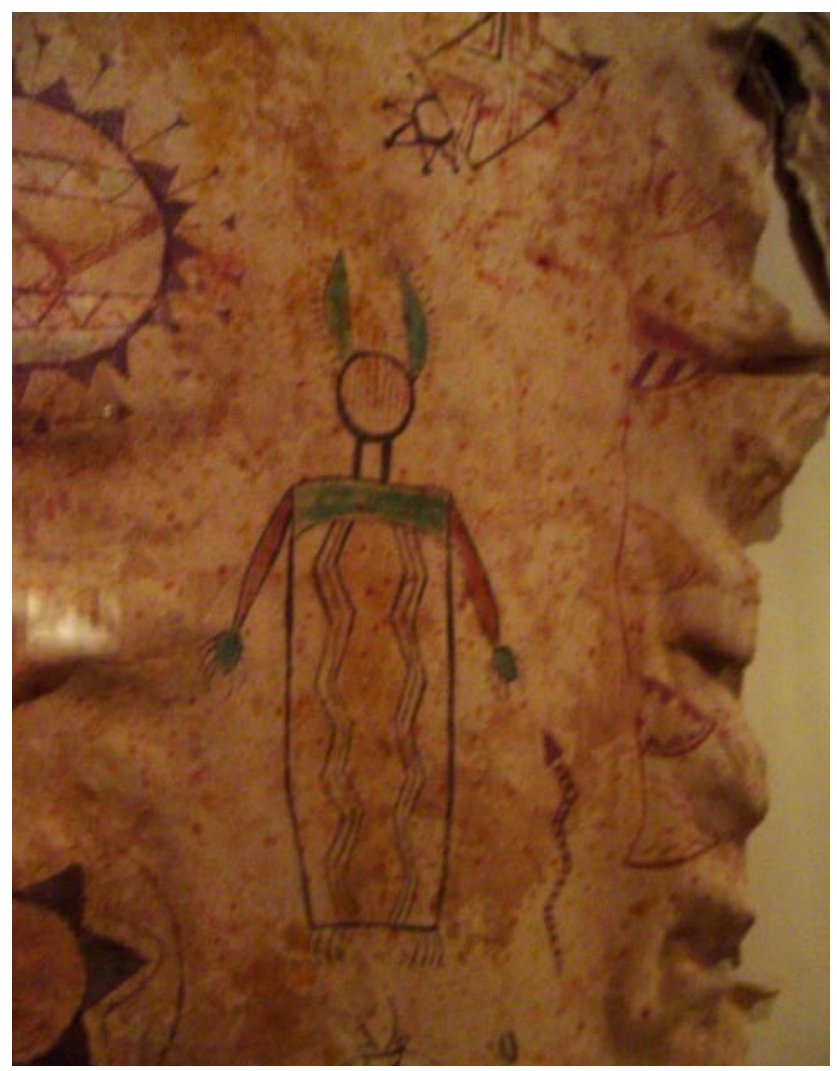

B. Representación humana en culturas de Norteamérica.

siderar esto como saber científico y pasarán miles de años para que la anatomía surja como ciencia. Estos conocimien- 


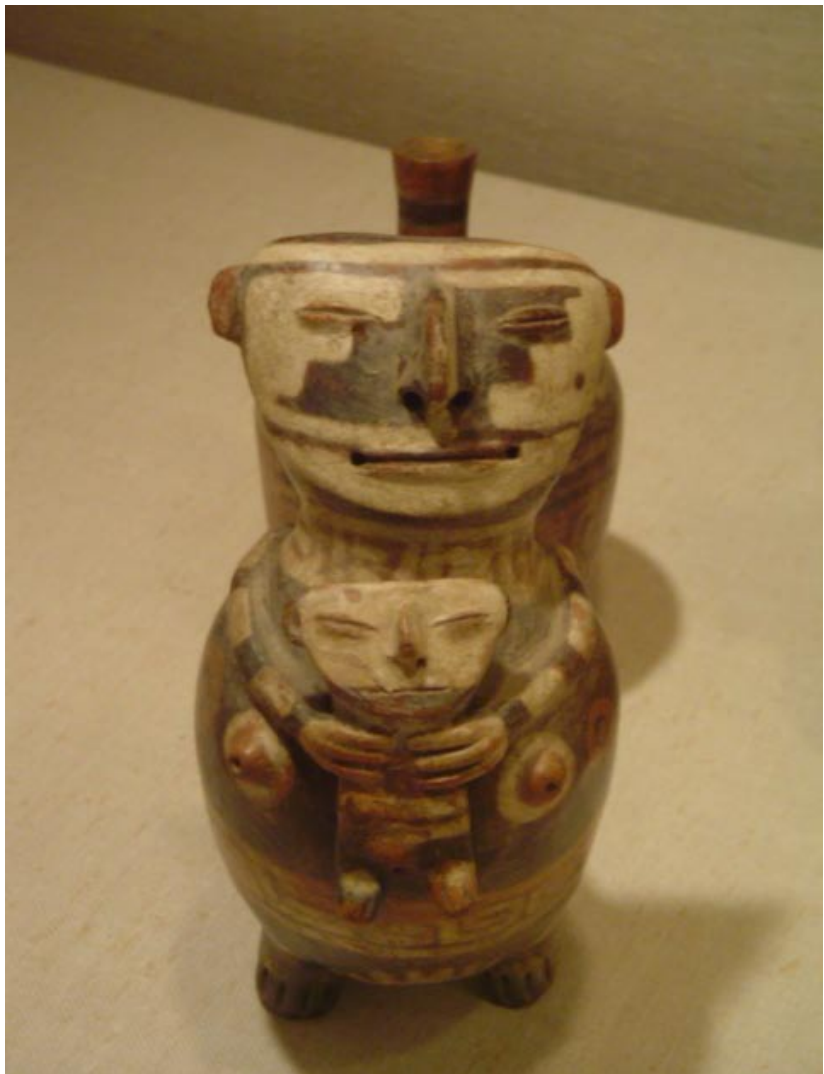

C. Figura de Arcilla que representa una madre y su hijo, culturas Africanas.

tos son inicialmente alegóricos y religiosos, su transmisión debe haber sido oral ya que salvo lo mencionado, no han quedado registros.

En la antigüedad, el avance del conocimiento anatómico se vio dificultado durante miles de años por problemas religiosos: estaba impedido abrir los cadáveres o incluso tomar contacto con ellos ${ }^{17}$. Pero además de ello, no se le daba demasiada importancia al sistema nervioso, ya que se consideraba que el centro de la vida y la espiritualidad se ubicaba en el corazón.

De forma breve se analizarán algunas culturas, en las cuales se hace mención a estructuras del sistema nervioso, lo que puede considerarse como los primeros antecedentes neuroanatómicos en la historia.

Los hindúes pueden haber disecado cadáveres de forma metódica hace más de 3000 años. Dichas disecciones debían ser llevadas bajo agua, para evitar que los cuerpos tomaran contacto con metal. En el Azour Veda, escrito por Brahma en el 1550 AC hay referencias a la circulación sanguínea $^{4,8,14,25}$.

En varias culturas se tienen registros de trepanaciones craneanas de más de 10.000 años de antigüedad: en el paleolítico en el actual Japón, en el Mesolítico en las actuales Alemania, Checoslovaquia, Hungría, Ucrania y
2008; 19: 58-65

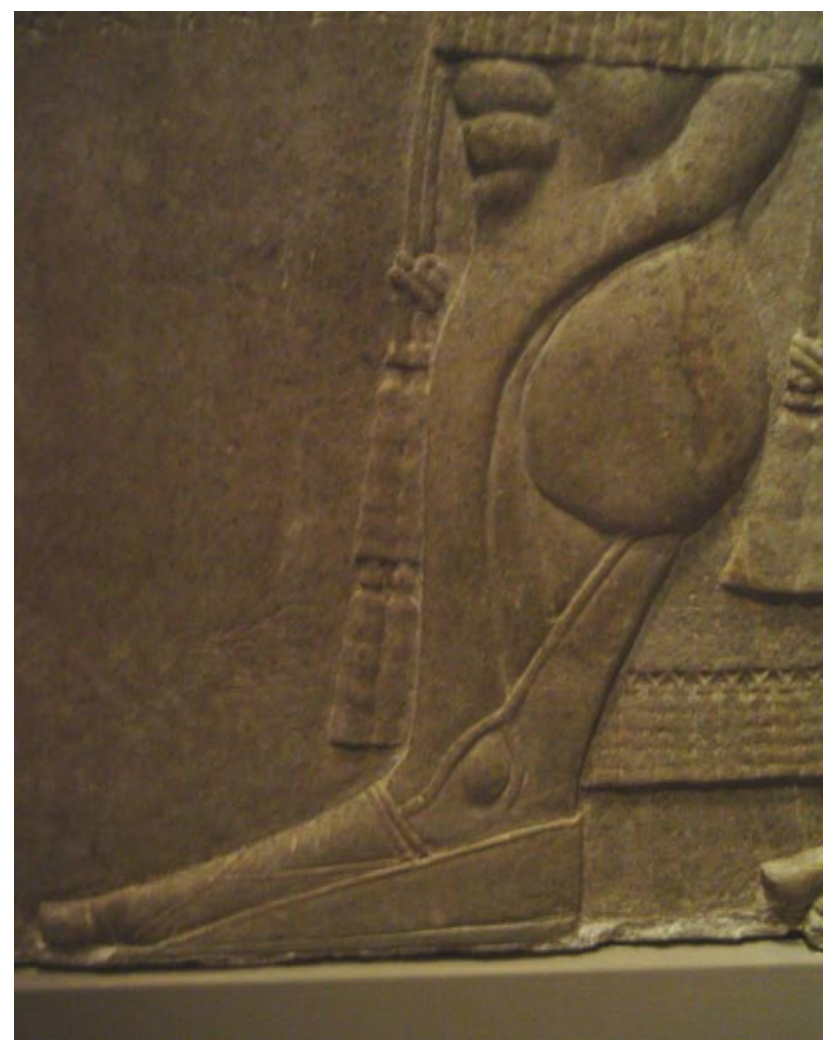

Figura 2. Explicación en el texto.

Península Ibérica, al igual que en América del Sur donde hay trepanaciones que datan de más de 3000 años AC. En muchos de estos cráneos se encontró hueso neoformado en los rebordes de la trepanación, por lo que los individuos sobrevivieron a la misma. Se plantea que estas trepanaciones tenían carácter terapéutico, para tratar hundimientos o dejar escapar los espíritus responsables de las

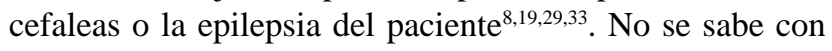
certeza si esto generó un conocimiento anatómico, pero sí es cierto que a través de estas craniectomías el hombre primitivo observó el cerebro o las meninges.

En la cultura mesopotámica (4000-540 AC), no se menciona al sistema nervioso, ya que: "El corazón es el centro de los movimientos del alma; el hígado el de las emociones sensibles; los riñones centro del vigor físico y el vientre el asiento de los sentimientos y la inteligencia"27. En la figura 2 se observa la vena safena interna en una escultura del 880 AC, hallada en el templo de Ashurnasirpal II, Nimrud.

En Egipto existió cierto saber, derivado de la observación de animales (3500 AC), heridas premortem, ritos funerarios, embalsamamiento y observaciones clínicas. El registro más antiguo de momificación corresponde a la momia de Hetep-Heres, madre de Keops (3400 AC). Es interesante destacar que durante el procedimiento de momificación, se extraían con gran delicadeza algunos órganos, como el corazón y los riñones, que se ubicaban en las jarras 


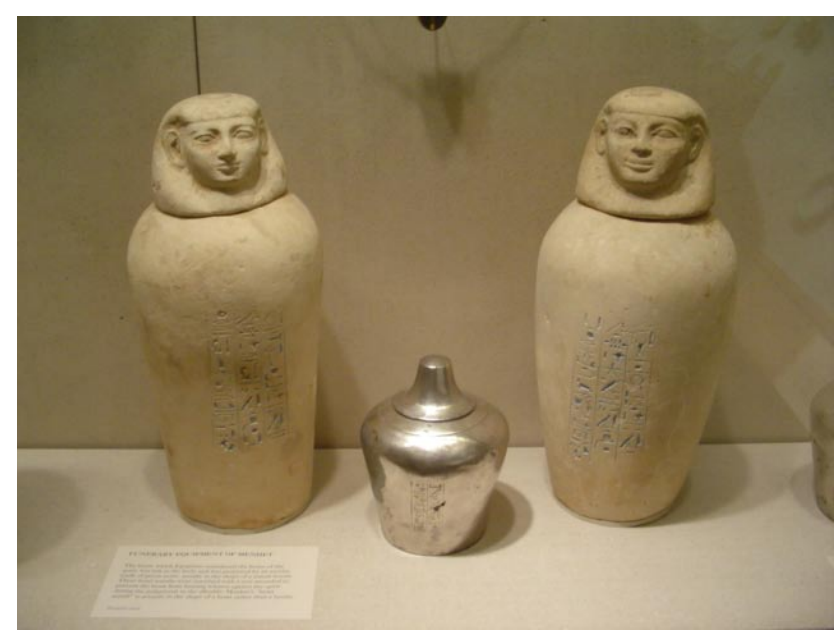

Figura 3. A. Jarros canópicos. Dentro de los mismos se colocaban órganos macizos, se observa en el exterior de los mismos las inscripciones de ofrenda a Horus.

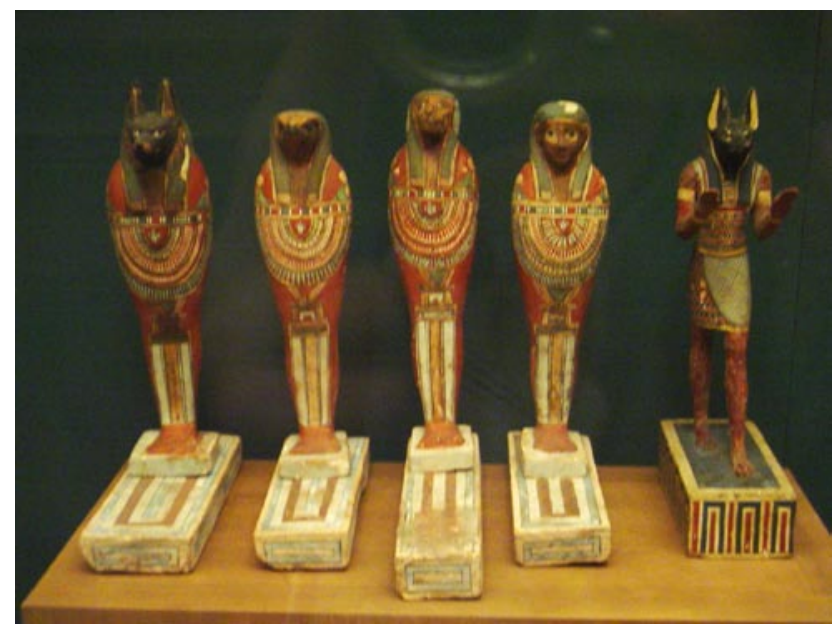

B. Figuras representativas de Anubis (a la derecha en la foto) y los cuatro hijos de Horus.

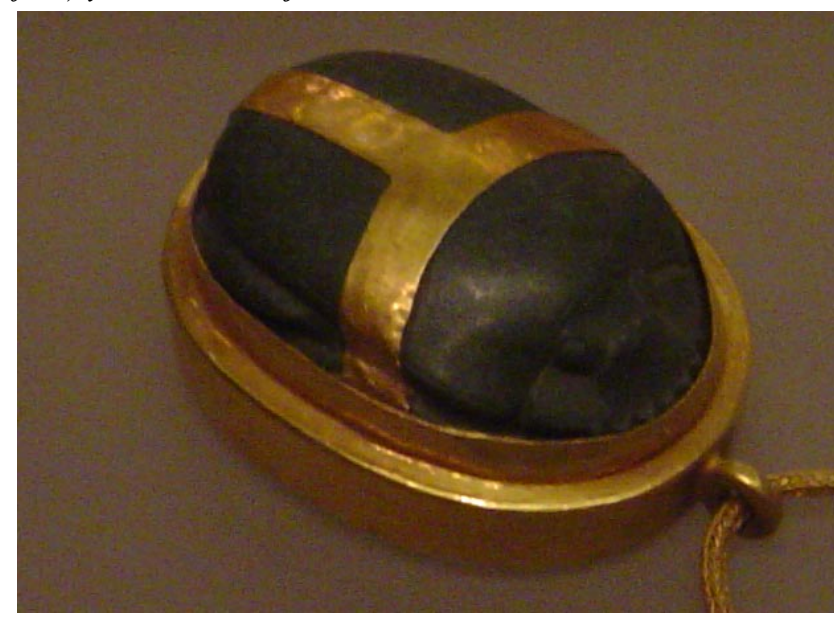

C. Detalle del escarabajo de piedra con encajes de oro que se colocaba en la posición del corazón durante el proceso de momificación.
2008; 19: 58-65

canópicas (Figura 3 a). El cerebro era extraído por las fosas nasales o a través de las cavidades orbitarias. Cada una de las jarras canópicas tenía un texto en su exterior, escrito en nombre de Anubis, diosa del embalsamamiento. Este texto estaba dirigido a los hijos de Horus (Imsety, Hapy, Kebehsenuef y Duamutef) para que protegieran los órganos allí contenidos (Figura 3 b). En el sitio donde se ubicaba el corazón, se colocaba un amuleto, en forma de escarabajo tallado en piedra verde y con adornos de oro (Figura $3 \mathrm{c}$ ).

El papiro de Edwin Smith fue escrito en el 1600 AC, si bien puede ser copia de uno aún mas antiguo. Es un tratado de medicina y cirugía; en él se mencionan las meninges, las circunvoluciones cerebrales y aparece por primera vez el término "cerebrum"3,4. En este texto se resumen 48 observaciones de lesiones del sistema nervioso, que se fundamentan en un conocimiento anatomofisiológico del mismo. Se menciona que una lesión en el cerebro produce imposibilidad en el movimiento de los miembros contralaterales, "...una herida en el cráneo da desviación de los ojos y el enfermo camina arrastrando el pie..."4. Los egipcios pensaban en la primacía del corazón sobre el resto de los órganos, pero éste parece ser el primer intento de atribuir al cerebro el papel rector del movimiento. Se describe también que al levantar la calota quedan al descubierto "... arrugas semejantes a las que se forman cuando se funde cobre...."4. Un enfermo con un trauma raquimedular "....está inconsciente de los dos brazos y las dos piernas, que su falo está en erección y que orina y eyacula sin saberlo..."4. "....Si examinas un hombre que tenga la sien hundida... no responde, ha perdido el uso de la palabra...", esta tal vez sea la mención mas antigua de una afasia.

No hay claros registros sobre la anatomía en el Imperio Persa y la Meseta Iraniana (2000 AC - 630 DC), pero varios términos usados por médicos árabes como Avicena (siglo XI DC), tienen origen en el antiguo iraní. Entre ellos interesa uno: Deilizi Medreb, derivado del término persa Dihliz, y que aparece en el Canon de Avicena para mencionar o definir un pasillo o corredor que comunica el ventrículo anterior (ventrículos laterales) con el posterior (cuarto ventrículo). Hyrtl en su texto anatómico cita el origen persa del término tercer ventrículo; se puede asumir que los persas conocieron, al menos en parte, el sistema ventricular cerebral ${ }^{8}$.

En la antigua India también hay registros de estructuras anatómicas. En el Rîgveda (himnos de transmisión oral y luego escrita) se lee "..De los ojos, de la nariz, de los oídos y del mentón - la consunción que existe en la cabeza te la expulso yo de la lengua y el cerebro...", formando parte del rito de expulsión de una deidad generadora de enfermedad ${ }^{8}$. El Corpus Médico de Sushruta (272 AC) menciona la existencia de 24 nervios y habla de disección cadavérica. Dice que debe elegirse el cadáver de un hombre no demasiado viejo o deforme y se describe como debe ser "vaciado 
de sus excrementos" para luego encerrarlo en un cajón, rodearlo de hierbas y sumergirlo en agua durante 7 días para "ablandar las carnes". Luego se frotaba el cadáver con un cepillo para hacer perceptibles los órganos internos.

El Corpus de Caraka (50 AC) describe la existencia de 900 nervios e indica los 7 puntos vulnerables o chakras, centros modificadores de la energía que se ubican en: 1) la base de la columna, 2) los genitales, 3) arriba del ombligo, 4) detrás del esternón, 5) el cuello 6) la glabela, 7) el bregma. Algunos autores llaman la atención sobre el paralelismo que existe entre los chakras y los plexos coccígeo, hipogástrico, pudendo, solar, cardíaco, cervical y faríngeo, así como la pineal y la corteza cerebral. Pero no podemos saber si esto se basó en un conocimiento anatómico verdadero, intuitivo o es casualidad.

Es interesante la concepción del macrocosmos hindú que tiene un paralelismo en la cultura griega, que a la postre influyó en la concepción de Galeno sobre los ventrículos cerebrales. Los hindúes planteaban tres elementos esenciales para la vida: Prâna (viento): es una fuerza material que genera movimiento y cambio, de él se origina el término nâdî, conductos por donde circula el prâna conectando los diferentes órganos entre sí. Describen tres nâdî principales alojados en la columna vertebral, y asociados a ellos los marman o centros neurovasculares.

a) Pitta (fuego): es la extensión del sol.

b) Kapha (agua): es la extensión de la luna y la luz lunar, es la materia común de todas las secreciones acuosas del organismo. Se subdividía en 5 entidades según el lugar del cuerpo donde reside la serosidad, de ellas el Snehana reside en la cabeza y permite las facultades sensoriales. ¿Se habrán referido los hindúes al líquido cefalorraquídeo?

En América, varias culturas realizaban sacrificios y ofrendas a sus dioses, que entre otras cosas, incluían la ofrenda de órganos de animales y humanos. No es claro si esto generó un conocimiento anatómico ya que la tradición escrita de muchas culturas fue destruida durante la conquista de América ${ }^{29,33}$.

\section{b) La antigua Grecia: filosofía, ciencia y anatomía}

La ciencia helena (siglos VI a IV AC) recibió influencias fenicias, hindúes y egipcias.

Antes del siglo VI AC la medicina griega era una mezcla de empirismo y magia; derivada de los templos de Asclepio ${ }^{14}$. Los médicos se originaron en castas que trasmitían los conocimientos de padres a hijos.

Pero entre los siglos VI y III AC se obtuvieron logros científicos geniales, fruto de un gran espíritu de debate que lleva a que las ciencias comienzan a desligarse de sus orígenes: la filosofía y la magia ${ }^{26,31,34-36}$.

En los siglos VI y V AC comienzan a plantearse conceptos más biológicos y "fisiológicos" de las enfermedades con lo que hace falta un mayor conocimiento anatómico.

Por ejemplo para Protágoras (490-420 AC) la fuente de conocimiento es la percepción de los sentidos ${ }^{16,17,19,26}$.

Demócrito de Abdera (460-370) plantea que todo está compuesto por una sustancia imperceptible a los sentidos: los átomos. Estos tienen diferentes formas y se combinan para originar todo; este pensamiento precursor fue combatido posteriormente por Aristóteles ${ }^{26}$.

Para Empédocles todo está constituido por 4 elementos básicos: agua, aire, fuego y tierra, lo que tiene un paralelismo en la cultura hindú, como se vio. Esta doctrina permaneció con pocos cambios hasta el siglo XVIII DC y en ella basa Galeno sus ideas fisiológicas ${ }^{26}$. Menciona además la presencia de aire en las arterias, el pneuma ${ }^{31}$.

Se piensa que en las escuelas de Crotona, Cirene y Cos (las más renombradas del siglo VI AC) se enseñaba semiología (semeión= mirar atentamente), farmacología, cirugía y anatomía basada en disecciones animales ${ }^{16,17}$.

Según Laín Entralgo ${ }^{16,17}$ con Alcmeon de Crotona comienza a gestarse una era revolucionaria dentro de la historia de la medicina: la medicina hipocrática, que se basa en la observación metódica y el razonamiento. En el 500 AC Alcmeon escribe el Peri physios, primer libro de medicina del que se tiene noticias según $\mathrm{Lasso}^{20}$, aunque como se mencionó, el papiro de Edwin Smith parece ser un tratado de cirugía y medicina ${ }^{23}$. Planteaba que la monarquía (predominio de uno de los humores) era causa de enfermedad $^{20}$. Para Alcmeon el hombre es un ser pneumático cuyas fuentes de vida son cuatro órganos: cerebro, corazón, ombligo y genitales. El cerebro es el centro de la vida y la inteligencia, no el corazón y el diafragma como se pensaba anteriormente $e^{4,19,20}$. Creía además que el esperma era parte del cerebro del varón desde los 14 años y que el alma existía, pero era algo material ${ }^{20}$.

Menciona los nervios ópticos y el quiasma, a los que cree huecos (los denomina póroi = canal, conducto) $\mathrm{y}$ afirma que las impresiones visuales y auditivas llegan al cerebro $^{20}$. Observó que alteraciones del cerebro producen cambios en la sensibilidad. Describe arterias y venas cerebrales y plantea que el sueño se debe a que el cerebro se vacía de sangre, que refluye hacia las venas; si la retirada de la sangre es total sobreviene la muerte, si esta regresa despertamos ${ }^{20,31}$.

Hipócrates de Cos, el padre de la Medicina occidental (460-370 AC) es una de las influencias más fuertes de Galeno. Escribió al menos 5 libros de anatomía: "Sobre la anatomía", "Sobre los huesos", "Sobre las glándulas", "Sobre las carnes" (músculos) y "Sobre el corazón". Para algunos autores estos textos pueden no haber sido escritos por Hipócrates y haber sido atribuidos a él posteriormente ${ }^{16-}$ ${ }^{19}$. En el "Corpus Hipocraticum" se mencionan estructuras encefálicas: menciona vasos cerebrales superficiales pero confunde arterias con venas, describe las meninges, 
"...una gruesa y otra delgada...", menciona que el cerebro se divide en dos mitades separadas por una membrana (la hoz del cerebro? $)^{16,17,30,31}$. Plantea que la fuente de la inteligencia estaba en el cerebro y que la lesión de un lado del cráneo puede dar una parálisis contralateral o trastornos del lenguaje ${ }^{11}$. Indicó la trepanación para las contusiones cerebrales y describió de forma excelente la clínica de la hemorragia subaracnoidea.

Es muy probable que Hipócrates no haya disecado cadáveres humanos dada la severidad religiosa de los griegos que enterraban o reducían a cenizas los cuerpos ${ }^{25,31}$. Se dio entonces excesiva ligereza al empeño de imaginar lo no visto y luego dar por cierto lo imaginado, esto fue la base de muchos errores ${ }^{36}$. Incluso la disección de animales debe haber sido escasa y fundamentalmente aprendida de las artes culinarias ${ }^{16,17}$.

Platón (428-348) plantea que la esfera es la figura perfecta: el alma debe estar entonces en la cabeza, que es redonda y está en lo más alto del edificio humano ${ }^{11}$. Como se verá en la segunda parte de este trabajo, Galeno utiliza esta concepción al referirse a la forma de los ventrículos cerebrales. Platón planteaba que el alma estaba separada del cuerpo y la dividía en tres tipos: 1) intelectual, la principal, situada en el cerebro, 2) sensitiva, ubicada en el corazón y 3) vegetativa, alojada en el abdomen superior ${ }^{9}$. Posteriormente Herófilo, ubicó cada una de estas facultades en los ventrículos cerebrales. Platón afirmaba que.. "El cerebro puede ser el origen de las percepciones de la audición, visión y olfato, y la memoria y la opinión pueden venir de ellas y la ciencia puede estar basada en la memoria y la opinión”.

Aristóteles (384-322 AC) funda la escuela peripatética ("que discute andando"), se lo considera el padre de la anatomía comparada ${ }^{11,26}$. Según Moncho $^{24}$ es el primero en utilizar la palabra "Anatome", término griego que significa corte, disección. No disecó cadáveres humanos, pensaba que lo observado en animales era verdadero en el hombre (analogía aristotélica). De todas formas no valía la pena la disección de cadáveres humanos ya que tiene el aspecto exterior de un hombre pero no lo son, ya que el neuma se ha ido ${ }^{1,11}$.

Menciona las meninges y parece ser el primero en mencionar que el cerebro posee cavidades $^{9,35}$. Pero da un paso atrás, revalorizando las funciones del corazón sobre las del cerebro. Este último es húmedo, frío e insensible no contiene otra cosa que sangre y su función es refrigerar y purificar la sangre que llega del corazón, órgano de las sensaciones, la inteligencia y el movimiento ${ }^{5,11,26,31}$. Aristóteles se basaba en sus observaciones de embriones de pollo, al ver la aparición temprana del esbozo cardíaco ${ }^{26}$. En Aristóteles está el origen de la teoría del pneuma ${ }^{11}$.

\section{c) La escuela de Alejandría, la ciencia helenística}

Las conquistas de Alejandro Magno permitieron una
2008; 19: 58-65

mezcla de culturas, pero su temprana muerte desintegra el imperio helénico originando nuevos focos culturales, entre los cuales brilla en el Egipto helenizado la ciudad de Alejandría ${ }^{26}$.

Se considera a Alejandría el centro cultural más importante de la antigüedad $d^{1,25,26}$. Esta escuela fundada alrededor del 323 AC por los Ptolomeos, es el primer centro de estudios de Occidente organizado y subvencionado por el Estado $^{1,5,11}$. Su biblioteca, fue la más grande de todas las que la precedieron e incluso de las que le siguieron por diecinueve siglos, ya que llegó a tener más de 500.000 libros $^{5}$. Allí se reunieron los tratados hipocráticos que constituyeron el Corpus y que reunía el conocimiento médico hasta el siglo IV AC ${ }^{5,26}$.

Alejandría puede ser considerada como la primera Universidad de la historia, la ciudad fue un centro de encuentro de culturas donde convivieron macedonios, judíos, egipcios, persas, hindúes, sirios y griegos, entre otros. Esta mezcla cultural logró que se derribara uno de los grandes obstáculos con los que chocó el aprendizaje anatómico: la prohibición de disecar cadáveres humanos ${ }^{5,17}$.

Aquí nace la Anatomía como ciencia, se realizó la disección metódica de cuerpos humanos en sitios especialmente destinados para ello. Por esta razón en Alejandrina encontramos la primera Escuela Anatómica ${ }^{25}$. Debe jerarquizarse que desde la posterior decadencia de Alejandría, no hay registros claros y confiables de disecciones de cadáveres humanos por cerca de 1.800 años ${ }^{1}$.

Se destacan Erasistrato de Kéos (330-250 AC) y Herófilo de Calcedonia (320-250 AC). Según Chapman ${ }^{5}$, si bien Erasistrato trabajó y estudió en Alejandría, desarrolló la mayor parte de su obra en Antioquia. Erasistrato describió los ventrículos y lo que es sumamente interesante: junto con Herófilo hablaron de que el número de circunvoluciones estaba en relación con la inteligencia humana.

Herófilo puede ser considerado el Padre de la Anatomía y su afición por la disección le valió el nombre de "devora muertos"; se piensa que disecó más de 600 cadáveres y se afirma que llegó a hacer vivisecciones en humanos ${ }^{1}$.

Entre otras estructuras describe las meninges, los ventrículos cerebrales, en particular el cuarto ventrículo donde observa el calamus scriptorius. Es el primero en describir los plexos coroideos y los senos venosos con la confluencia de los senos (prensa de Herófilo) ${ }^{1,5,11,30,31}$. Es además el primero en mencionar la rete mirabile, que sería mejor descrita por Galeno.

Erasistrato y Herófilo plantean la teoría ventricular. En la misma describen el pneuma, sustancia que se forma y purifica en el cerebro y el corazón viajando por los nervios hacia los músculos. El principio de la actividad está en el pneuma animal alojado en los ventrículos, que se forma del pneuma vital que viene del corazón. El pneuma animal viaja desde los ventrículos laterales hacia un ventrículo 
común (tercer ventrículo), que comunica con el ventrículo ubicado debajo del cerebelo (cuarto ventrículo). En este trayecto se purifica para luego ingresar a los nervios. A través de los nervios motores, duros viaja el pneuma psíquico hacia los músculos ${ }^{11}$. Para Foulon ${ }^{11}$, Herófilo y Erasistrato confundieron los tendones con nervios, sin embargo para otros autores, Herófilo es de los primeros en reconocer la diferencia entre nervios y tendones e incluso diferenciaron nervios sensitivos de motores ${ }^{5}$. Más allá de esta discusión, esta circulación del pneuma desde el cerebro a través de los nervios, hacia los músculos, podría ser considerado como un esbozo de neurotransmisión.

Rufus de Éphese (110-180 DC), maestro de Galeno, toma clases de anatomía en Alejandría. Distinguió cerebro de cerebelo, hizo una detallada descripción de las meninges, ventrículos laterales, cuarto ventrículo, acueducto de Silvio y tercer ventrículo, hipófisis, placa cuadrigeminal, glándula pineal, cuerpo calloso y trígono.

Esta es entonces, la base cultural que sirvió a Galeno para plantear sus teorías anatómicas. Puntualmente se verá la concepción anatomofuncional de Galeno sobre los ventrículos cerebrales en la segunda parte de este trabajo.

\section{Agradecimientos}

Los autores desean agradecer especialmente a Sandra Burgues Roca (Departamento de Historia de la Medicina, Facultad de Medicina de Montevideo, Uruguay) y al Prof. Emérito Dr. Fernando Mañe Garzón, Director del Departamento de Historia de la Medicina. El Profesor Mañé Garzón nos dedicó de forma generosa y desinteresada su tiempo, nos permitió acceder a la invalorable biblioteca de su Departamento y donó al Departamento de Anatomía textos históricos.

\section{Bibliografía}

1. Acar, F., Naderi, S., Guvencer, M., Türe, U., Arda, M.N.: Herophilus of Chalcedon: a pioneer in neuroscience. Neurosurgery 2005; 56: 861-867.

2. Apuzzo, M.L.J.. The legacy of Galen of Pergamon. Neurosurgery 2000; 47: 545.

3. Brieger, G.H.: Desarrollo de la cirugía. Aspectos históricos sobre el origen y la evolución de la ciencia quirúrgica moderna. En: Sabiston DC (ed.) Tratado de patología quirúrgica. Interamericana, México, 1991; pp.1-20.

4, Changeux, J.P.: El hombre neuronal. Editorial EspasaCalpe S.A., Madrid, 1985.

5, Chapman, P.H.: The Alexandrian library: crucible of a renaissance. Neurosurgery 2001; 49: 1-14 (ver comentarios).

6, Cruz Hernández, M.: Visión sinóptica de la cultura islámica (los principios fundamentales de la cultura musulmana). En: Laín Entralgo, P. (ed.): Historia universal de la medicina.
Tomo III, Salvat Editores, Barcelona, 1972; pp. 41-58.

7. Daremberg, Ch.: Oeuvres anatomiques, physiologiques et médicales de Galien. J B Bailière, Paris, 1854; pp. 524608.

8. Decuadro, G., Martínez, F., Mañé Garzón, F., Burgues Roca, S.: El conocimiento neuroanatómico previo a Galeno. Presentado en II Jornadas de enseñanza de la Anatomía de la Sociedad Argentino Uruguaya de Anatomía. Buenos Aires, Argentina, Setiembre de 2005.

9. Del Maestro, R.F.: Leonardo da Vinci: the search for the soul. J Neurosurg 1998; 89: 874-887.

10. Foote, R.R.: Venas varicosas. López Libreros Editores, Buenos Aires, 1969; pp. 17-50.

11. Foulon, P.: Histoire des ventricules cérébraux. Neurochirurgie 2000; 46: 142-146.

12. Goodrich, J.T.: Landmarks in the history of neurosurgery. In: Rengachary S, Wilkins R (ed.) Principles of neurosurgery. Mosby-Wolfe, Hong Kong, 1994; pp. 1-13

13. García Ballester, L.: Galeno. Ed. Guadarrama/Punto omega. Madrid, 1972; pp. 73-165.

14. Hahn, A., Goichon, A.M.: Le decouverte de la circulation pulmonaire par un médicine arabe au XIII ${ }^{\text {eme }}$ siècle. Presse Med 1952; 60: 191.

15. Kudlien, F.: Medicina Helenística y Helenísticoromana (300 a.c.-100 d.c.).En: Laín Entralgo, P. (ed.): Historia universal de la medicina.Tomo II La antigüedad clásica. Salvat Editores, Barcelona, 1972; pp. 153-200.

16. Laín Entralgo, P:. La medicina Hipocrática. Ediciones de la Revista de Occidente, Madrid, 1970; pp. 1-30.

17. Laín Entralgo, P.: La medicina Hipocrática. En Laín Entralgo, P. (ed.): Historia universal de la medicina. Tomo II La antigüedad clásica. Salvat Editores, Barcelona, 1972; pp. 73-118.

18. Laín Entralgo, P.: ¿Conoció Galeno la circulación de la sangre? Med Clín 1946; 7: 464-466.

19. Laín Entralgo, P., García Ballester, L.: Medicina bizantina. En: Laín Entralgo, P. (ed.): Historia universal de la medicina. Tomo III, Salvat Editores, Barcelona, 1972; pp. 9-40.

20. Lasso de la Vega, J.S.: Pensamiento presocrático y medicina. En Laín Entralgo, P. (ed) Historia universal de la medicina. Tomo II La antigüedad clásica. Salvat Editores, Barcelona, 1972. pp. 37-65.

21. Marino, R. Jr., Niemeyer, P., Bello, H.: Histórico da cirurgia da epilepsia. En: Costa da Costa, J., Palmini, A., Targas Yacubian, E.M., Cavalheiro, E. (eds.) Fundamentos neurobiológicos das epilepsias: aspectos clínicos e cirúrgicos. Lemos Editorial, Sao Paulo, 1998; pp. 1045-1058.

22. Martínez, F., Armand Ugón, G., Laza, S., Sgarbi, N.: Estudio de los ventrículos cerebrales mediante inyección de resinas poliéster. Rev Neurocirugía (La Plata) 2006; 8: 18-21.

23. Martínez, F., Decuadro, G., Burges, S., Mañé Garzón, F.: Claudio Galeno y el sistema ventricular encefálico. Presentado en II Jornadas de enseñanza de la Anatomía de la 
Sociedad Argentino Uruguaya de Anatomía. Buenos Aires, Argentina, Setiembre de 2005.-

24. Moncho, R.: Anatomía funcional del sistema venoso de los miembros inferiores y su transferencia a la clínica. Libro de resúmenes del XXXIII Congreso de la Asociación Rioplatense de Anatomía, Corrientes, Argentina, 1996; pp. 9-29.

25. Olóriz Guerra, D.: Manual de técnica anatómica. El Cosmos Editorial, Madrid, 1890; pp. 4-13.

26. Papp, D.: La ciencia en el mundo grecorromano. En: Laín Entralgo, P. (ed.): Historia universal de la medicina. Tomo II, Salvat Editores, Barcelona, 1972; pp. 17-36.

27. Porter, B.A.: Art of the ancient near east. LS Graphic, Italia, 2002: 6 pp.

28. Premuda, L.: Anatomía en la baja edad media. En: Laín Entralgo, P. (ed.): Historia universal de la medicina. Tomo III, Salvat Editores, Barcelona, 1972; pp. 297-312.

29. Rifkinson-Man, S.: Cranial surgery in ancient Perú. Neurosurgery 1988; 23: 411-416.

30. Ríos Bruno, G.: Bosquejo de historia de la anatomía universal. Rev Hospital Maciel $2^{a}$ Época 1997; 1: 41-48.

31. Salamon, G.: Atlas de la vascularisation arterielle du cerveau chez l’homme. Sandoz Editions, Paris. 1971.
32. Schiepperges, H.: La medicina en el medioevo árabe. En Laín Entralgo, P. (ed.): Historia universal de la medicina. Tomo III, Salvat Editores, Barcelona, 1972; pp. 59-118.

33. Solís, F.: The aztec empire. Catalogue of the exibition. Finlay Printing, Connecticut, 2004: 80 pp.

34. Tovar, A.: Sinopsis de la antigüedad clásica. En Laín Entralgo, P. (ed.): Historia universal de la medicina. Tomo II, Salvat Editores, Barcelona, 1972; pp. 1-17.

35. Walker, A.E.: The cerebrospinal fluid from ancient times to the atomic age. En: Walker A.E., Arana-Iñiguez, R. (eds.) Cerebrospinal fluid in health and disease. Acta Neurol Latinoamer 1971; 17(Suppl 1): 1-10.

36. Whitehead, A.N.: Science and the modern world. The Macmillan Company, New York, 1948.

Martínez, F.; Decuadro-Sáenz, G.: Claudio Galeno y los ventrículos cerebrales. Parte I, los antecedentes. Neurocirugía 2008; 19: 58-65.

Correspondencia postal: Dr. Fernando Martínez. Departamento de Anatomía. Facultad de Medicina. Gral. Flores 2125 esquina Yatay, Montevideo, Uruguay. 